\title{
The Construction of 3D Virtual Camera Laboratory Based on Virtools
}

\author{
Ling HE
}

\author{
Jiangxi Science and Technology Normal University, Nanchang JiangxiProvince, China \\ lynlynhe126@126.com
}

Keywords: Virtual experiment, Virtools, Design, Development.

\begin{abstract}
Virtual reality technology (Virtual Reality, referred to as VR) is the latest technology in a appeared in the late nineteen eighties in the computer field, and has become a hot topic in education application. It will play a positive role in improving the students' skills and saving hardware investment . In this paper, the use of Virtools as a development tool of virtual reality, the camera as an example, the overall framework design flow chart of a virtual experiment is proposed, on each link and functions are discussed in detailed design, build a camera virtual experiment, discusses the key technology of 3D modeling and experimental interaction function and Realization method.
\end{abstract}

Virtual reality technology, also known as virtual reality technology, which is based on computer technology, use and integrated 3D graphics technology, multimedia technology, simulation technology, sensor technology, display technology and other high-tech latest development achievement, to generate a virtual world forced 3D vision, tactile, olfactory and other sensory experience using computers and other equipment, so that the virtual world produce a kind of the scene feeling for a person. Virtual reality and network, multimedia technology which are known as the three major technology with the application prospect of $2 \mathrm{l}$ century, and VR has been widely used in the military exercise, sports training, medical, commercial and other fields.

Virtual Laboratory (Virtual Laboratory) is also known as the "cooperative Laboratory (Collaboratory)", used to describe a virtual lab environment under the computer network environment, and proposed in 1989 by Professor William WuIf Virginia of the University of the United States of America. It can replace the related hardware and software of traditional experimental operation and operating environment, make the user finish all kinds of experimental projects like in the real environment, and effectively reduce the cost, improve the efficiency, and even also can realize collaborative and experimental resource sharing through the network.

\subsection{The Situation of Camera Instruction}

The teaching course of the camera can not be just as one wishes. Facing the camera, digital technology change rapidly development, we still use rigid, outdated teaching content and teaching method; high-speed development in the digital camera today, camera equipment updating, post processing software and image output products emerge in an endless stream of late in general colleges and universities, can only meet the investment professional basic teaching equipment, has been unable to fit the needs of professional teaching, and school education and social development, the market gap phenomenon; teachers generally use text, pictures, video mode of teaching, students cannot participate in practice, obtain perceptual knowledge; students scale expands, but learning time is not enough, teaching in practice often people form a group, the common camera equipment, careful observation, students lack experience and digestion time. These are serious constraints in the practice of cultivating the ability to operate the experiment teaching quality improvement, students.

By constructing a virtual laboratory can make learning not affected by time, place restrictions, experimental operation, and familiar with the camera function, understand the structure and operation method, the camera shooting skills practice, master the shooting light and picture composition skills, get a visual, real effect. Not only does this create good conditions for self-taught learners, so that they 
can learn at their own level and ability, but also can effectively cultivate students' practical ability, operation ability, achieve teaching objectives.

\subsection{Development Tool - Virtools}

Virtools is the global interactive 3D development under by the French Dassault group solutions developed, which is widely used in the architectural design, game development, industrial simulation, virtual training and so on, can publish works to PC personal computer, a game console, LAN and the Internet, to share the 3D experience to the general population more. Virtools includes five key components: GUI (Graphical User Interface, interactive graphical user interface), with a visual editing mode, the flow chart of the mode of thinking, objects and script design work, effectively shortens production cycle; Behavior Engine (script engine) to run interactive applications; Render Engine (rendering engine) to display graphic images in real-time rendering method; VSL (Virtools Scripting Language, a scripting language) in the code, programming, a part of the optimization of script, improve efficiency; SDK (software development kit, a software development kit) used to create custom scripts and applications, as well as the function of Virtools the expansion.

Virtools provides rich interactive module (Building Blocks, referred to as BB), with the direct drag way, BB is assigned to the appropriate Object (object) or Character (role), and in the script (script) edit in the form of a flowchart, thus forming a complete virtual interactive world.

\subsection{Construciton of 3D Virtual Laboratory Based on Virtools}

The development process of 3D virtual experiment is the use of computer virtual reality technology and product modeling technology as the foundation, the development process to complete the experiment by computer. One of its main features is the use of computer generated virtual equipment instead of physical simulation experiment.

The virtual experiment is made up for two-dimensional interface and 3D scene. Two-dimensional interface is mainly used to beautify the experimental background, placing a control button, display data and other information. The 3D scene is the main part of the system, through the design of interactive script, interactive virtual experiment. As shown in figure1. 3.1.

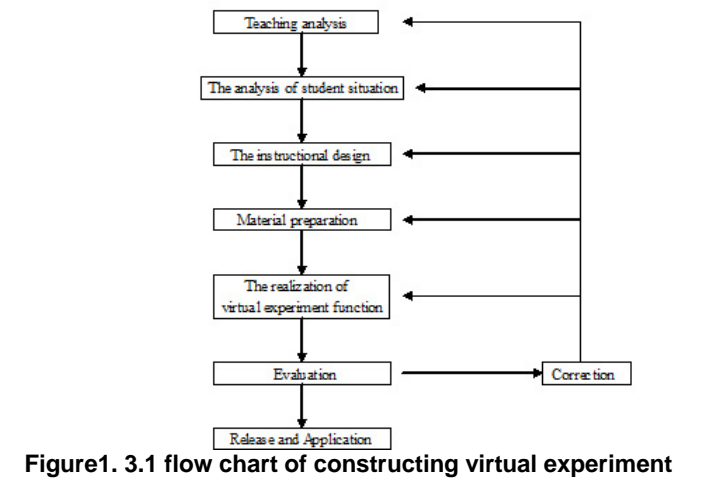

Before manufacture, we should make clear the teaching request, collect the related information, including the position and role of the curriculum, teaching objective, teaching content, teaching requirements and teaching emphasis and difficulties. Analysis of feasibility and necessity of the development of virtual experiment is suitable for analysis of the development of virtual experiment.

Analysis of the Student situation is also called analysis of learners.It is the key of virtual experiment design, experiment content should focus on the learner, this is also a kind of "learner-centered" teaching thought. Analysis of learners is to understand the preparation and style of learning , thus, the educators can teach students in accordance with their aptitude and the students will become a prepared learners.

According to the characteristics of teaching goals and learners, it is need to choose and organize rationally teaching media and teaching methods to form teaching system structure optimization. 
According to the teaching goal,using the concept and method of system theory, can analyze the problems and needs in teaching process, and identify the steps to solve the problem.

According to the virtual experiment design, material preparation includes images, text, sound, and video etc, by using software we can deal with these materials. Image file format support: JPG, PNG, TIFF, TGA, BMP, PCX. The sound file formats support: MP3, WMA, WAV, MIDI. And the camera is modeled, as is shown in fig1.3.2..

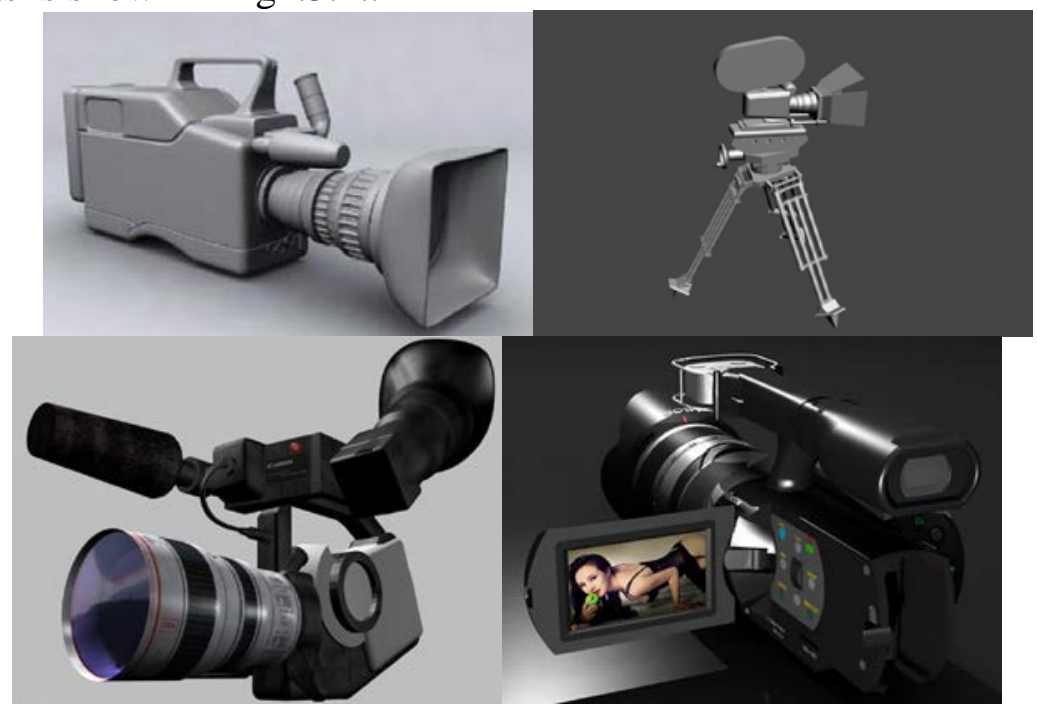

Fig. 1.3. 2 The 3D models of Digital Video Camera

Using Virtools to design interactive script, which is the key of development process. We can use BB module owned byVirtools to make all kinds of basic operations on 3D or 2D model, such as rotation, translation, scaling, color changes, changes in light, explosion effect,we can also add text and sound. In addition to the use and repeated use of BB module combined with Virtools , we can also manual automatically write $\mathrm{BB}$ by using $\mathrm{C}++$, and gradually make it a complete virtual experiment system.

Take behavior module which simulates camera aperture rotation as an example, the behavior module of Wait Message waits for a mouse click message, after a mouse click the behavior will be activated. By triggering Sequencer sequence generator module and determining the direction of rotation of the selector aperture Parameter selector Parameter Selector, designated in accordance with different imput will output corresponding parameters, the behavioral modules of Bezier Progression is to calculate a floating-point number between 2D Baez curve of the minimum and maximum range . and jointly control the direction of rotation of the aperture with Identity、Multiplication. Rotate behavior module performs rotary entity function. As is shown in the Figure Fig.1.3.3. After filming, it is need to play video, whose function module settings, is shown in the image Fig 1.3.4:

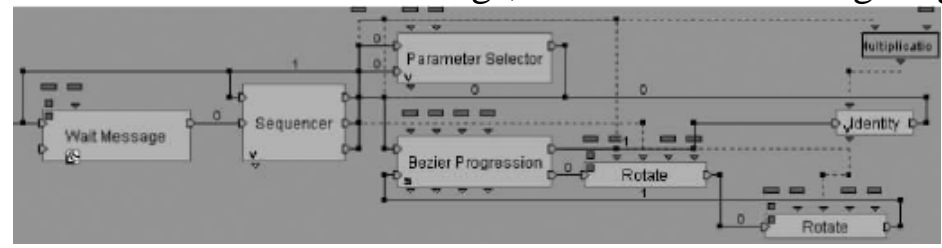

Fig. 1.3.3 aperture rotation behavior module

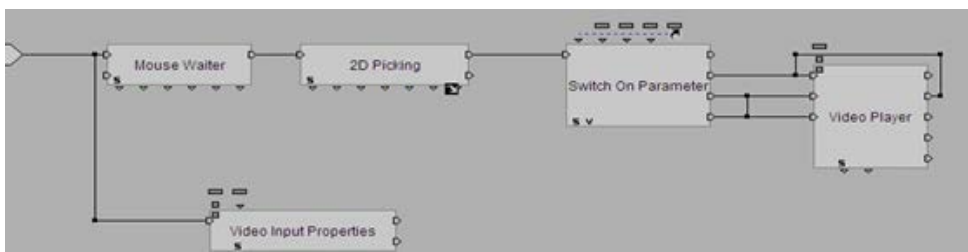

Fig. 1.3.4 video playback behavior module 
In the production process of virtual experiment,it is necessary to constantly evaluate the system and modify the work to find out the mistakes and shortcomings of the program ,and make constantly improvement and optimization, which is an important part of virtual experiment in the manufacturing process, and quality assurance.

According to the need,we choose the release forms, one is through the Export to Virtools Player, to output.Vmo file, this issue form can play by installing the Virtools Web Player plug-in. But if they are relatively large documents, we can directly use Virtools Make Exe builder to make it as Exe application file.. Another option is to choose the Create Web Page command in the file to export the file as HTML webpage format.

\subsection{The prospect of 3D Virtual Teaching Laboratory based on Virtools}

The experiment is an important link in the teaching process, the development is directly related to the students' skill level. The use of virtual laboratory teaching can effectively solve the shortage of funds, backward teaching teaching equipment problems, and study will benefit the development of dangerous, difficult to operate in the laboratory. With the development of virtual technology and the application of stereo projection technology in the teaching field, Virtools can use Vrpack to stereo projection the released the virtual experiment teaching system works onto the screen. Compared with the traditional teaching model,it has the image, intuitive, immersion, the quality and efficiency of teaching has greatly improved. At the same time, the use of the combination of virtual reality technology and network has a broad space in distance education, it is a new development direction in the future experiment teaching.

\section{References}

[1] Hu Xiaoqiang, the virtual reality technology basic knowledge and application [M]. Beijing:

Beijing University of Posts and Telecommunications press, 2009

[2] Fu Zhiyong, Gao Ming. The 3D game design [M]. Beijing: Tsinghua University press, 2008

[3] Liu Mingkun. Three-dimensional game designer collection: Virtools development tools [M].: Sichuan Sichuan electronic audio-video publishing house, 2005

[4] Wang Liqun, Li Hongsong. The computer game planning and Design: Virtools simple tutorial [M]. Shanghai: Fudan University press, 2008

[5] Liu Mingkun. Three-dimensional game designer collection: Virtools game programming [M]. Guangdong: Shantou University press, 2006

[6] Liu Mingkun.VT game creation: [M]. Beijing: China Youth Press, 2010

[7] Runlina virtual experiment of Virtools research and design based on computer hardware in school [D]. Sichuan Normal University, 2012

[8] Liwei. 3D virtual laboratory research and Implementation Based on Virtools [J]. experimental technology and management, 2012, 27 (5): 83-86.

[9] Xu Yingxin, Wang Dandong, Xu Lin. 3D game designer collection [M]. Chongqing: computer electronic audio-video publishing house, 2009 